\title{
On the Performance Analysis of Equal-Gain Diversity Receivers over Generalized Gamma Fading Channels
}

\author{
Nikos C. Sagias, Member, IEEE, George K. Karagiannidis, Senior Member, IEEE, \\ P. Takis Mathiopoulos, Senior Member, IEEE, and Theodoros A. Tsiftsis, Member, IEEE
}

\begin{abstract}
A versatile envelope distribution which generalizes many commonly used models for multipath and shadow fading is the so-called generalized Gamma (GG) distribution. By considering the product of $N$ GG random variables (RV)s, novel expressions for its moments-generating, probability density, and cumulative distribution functions are obtained in closed form. These expressions are used to derive a closed-form union upper bound for the distribution of the sum of GG distributed RVs. The proposed bound turns out to be an extremely convenient analytical tool for studying the performance of $N$-branch equalgain combining receivers operating over GG fading channels. For such receivers, first the moments of the signal-to-noise (SNR) at the output, including average SNR and amount of fading, are obtained in closed form. Furthermore, novel union upper bounds for the outage and the average bit error probability are derived and evaluated in terms of Meijer's G-functions. The tightness of the proposed bounds is verified by performing comparisons between numerical evaluation and computer simulations results.
\end{abstract}

Index Terms-Equal-gain combining (EGC), generalized fading channels, generalized Gamma, Lognormal, Nakagami- $m$, outage probability, sum of random variables, Weibull.

\section{INTRODUCTION}

A GENERAL envelope distribution which includes many well-known channel models for both multipath as well as for shadow fading is the so-called generalized Gamma (GG) distribution. This distribution was introduced by Stacy, back in 1962, as a generalization of the (two-parameter) Gamma distribution [1] and it includes the Rayleigh, Nakagami-m, and Weibull as special cases, while it can also describe the Lognormal as a limiting case. Interestingly enough, despite its ability to characterize so many different fading channel

Manuscript received April 27, 2005; no revision; accepted August 22, 2005. The editor coordinating the review of this paper and approving it for publication is Dr. R. M. Buehrer. This paper was presented in part at the $2^{\text {nd }}$ IEEE International Symposium on Wireless Communication Systems (ISWCS), Siena, Italy, Sept. 2005.

N. C. Sagias was with the Institute for Space Applications and Remote Sensing, National Observatory of Athens, Metaxa \& Vas. Pavlou Street, Palea Penteli, 15236 Athens, Greece. Now he is with the Institute of Informatics and Telecommunications, National Centre for Scientific Research - "Demokritos," Agia Paraskevi, 15330 Athens, Greece (e-mail: nsagias@ieee.org).

P. T. Mathiopoulos and T. A. Tsiftsis are with the Institute for Space Applications and Remote Sensing, National Observatory of Athens, Metaxa \& Vas. Pavlou Street, Palea Penteli, 15236 Athens, Greece (e-mail: mathio@space.noa.gr, tsiftsis@ieee.org).

G. K. Karagiannidis is with the Division of Telecommunications, Department of Electrical and Computer Engineering, Aristotle University of Thessaloniki, GR-54124 Thessaloniki, Greece (e-mail: geokarag@auth.gr). Digital Object Identifier 10.1109/TWC.2006.05301. models, only very recently the topic of performance analysis of digital receivers over this generalized channel has gained renewed interest. Particularly, in an early work on this topic [2], Coulson et al. presented expressions in the form of infinite series for the average bit error probability (ABEP) of singlebranch receivers operating over a GG fading environment, with binary phase-shift keying (BPSK) and binary frequencyshift keying (BFSK) modulations. In another related work [3], Yacoub introduced the $\alpha-\mu$ distribution and gave a physical justification for the origin of the GG model. More recently, Aalo et al. presented a closed-form expression for the ABEP for both coherent and noncoherent/differentially coherent binary digital modulations [4].

The performance of diversity receivers has been extensively studied in past for the most important fading channel models, Rayleigh, Nakagami-m, and Weibull (e.g. see [5]-[8]). However, a performance study of diversity and specifically equalgain combining (EGC) receivers over GG fading channels has not been presented yet ${ }^{1}$. The main difficulty in studying EGC receivers is that the distribution of the sum of fading envelopes is required. The derivation of this distribution in terms of tabulated functions is a very difficult task [5]. Concerning this well-recognized but cumbersome statistical problem, several approaches aiming at providing possible solutions have been published in the open technical literature. In possibly one of the earliest works, Stacy in his original GG paper developed an infinite series approach for determining the cumulative density function (cdf) of the sum of GG distributed random variables (RV)s [1]. Many years later, in an approach independent from that in [1], Beaulieu derived an infinite series for determining the cdf of the sum of Rayleigh distributed RVs [10]. Helstrom has computed the distribution of such a sum using saddlepoint integration for uniformly weighted RVs [11], as well as for arbitrary weights [12]. Filho and Yacoub in [13] have derived an approximate probability density function (pdf) expression for the sum of Nakagami- $m$ RVs. Very recently, $\mathrm{Hu}$ and Beaulieu have presented accurate and simple closed-form approximations to the cdf and pdf of the sum of independent and identically distributed (i.i.d.) Rayleigh RVs [14], while

\footnotetext{
${ }^{1}$ After our paper has been accepted for publication, we became aware of another independent from our work contribution [9], which also address the problem of analyzing the error rate performance of EGC receivers over generalized Gamma fading channels. However, in [9] the solutions proposed are presented in integral form, whereas our approach involves closed-forn bounds.
} 
Karagiannidis et al. have presented a closed-form union upper bound for the cdf of the weighted sum of $N$ independent Rayleigh RVs [15]. All in all, although the problem of finding the distribution of the sum of fading envelopes has been extensively studied in the past for various distributions, the majority of the published methods being approximate solutions usually involving a truncation error. Hence, the derivation of an exact solution, in terms of tabulated functions, even for the simplest Rayleigh distribution, when $N>2$ and with nonidentical statistical parameters, still remains an open research problem.

In this paper, in an effort to provide a solution to this problem and within the framework of studying the performance of EGC receivers over GG fading channels, another approach is proposed. Since an analytical solution for the distribution of the sum of RVs is very difficult to derive, the use of union bounds is proposed. In particular, by deriving a useful expression for the cdf of the product of $N$ GG RVs and based on a well-known inequality between arithmetic and geometric means, closed-form union upper bounds for the cdf of the sum of GG distributed RVs are obtained. These bounds, which turn out to be quite tight, are used to analyze the ABEP and outage performance of $N$-branch EGC receivers operating over GG fading channels.

The remainder of the paper is organized as follows. In Section II, various statistical characteristics of the GG distribution are provided, while formulae for the distribution of the product as well as an upper bound for the cdf of the sum of $N$ GG fading envelopes are presented. In Section III, various performance criteria of EGC receivers operating over GG fading channels are obtained, while in Section IV, numerical and computer simulation results are presented and compared. Finally, useful concluding remarks are provided in Section V.

\section{Statistics of the GG Distribution}

Let us consider $N \geq 1$ independent three-parameters' GG distributed RVs $\left\{R_{\ell}\right\}_{\ell=1}^{N}$ with pdf given by [1, eq. (1)]

$$
f_{R_{\ell}}(r)=\frac{\beta_{\ell} r^{m_{\ell} \beta_{\ell}-1}}{\left(\Omega_{\ell} / m_{\ell}\right)^{m_{\ell}} \Gamma\left(m_{\ell}\right)} \exp \left(-\frac{m_{\ell}}{\Omega_{\ell}} r^{\beta_{\ell}}\right)
$$

where $\beta_{\ell}>0$ and $m_{\ell} \geq 1 / 2$ are two parameters related to the fading severity, $\Omega_{\ell}$ is related to the average fading power as $\mathcal{E}\left\langle R_{\ell}^{2}\right\rangle=\left(\Omega_{\ell} / m_{\ell}\right)^{2 / \beta_{\ell}} \Gamma\left(m_{\ell}+2 / \beta_{\ell}\right) / \Gamma\left(m_{\ell}\right)$, with $\mathcal{E}\langle\cdot\rangle$ denoting expectation, and $\Gamma(\cdot)$ being the Gamma function $[16$, eq. (8.310/1)]. The distribution in (1) is very generic ${ }^{2}$ since it includes commonly used fading models such as Rayleigh (for $\beta_{\ell}=2$ and $m_{\ell}=1$ ), Nakagami- $m$ (for $\beta_{\ell}=2$ ), and Weibull (for $m_{\ell}=1$ ) as special cases. Moreover, for the limiting case of $\beta_{\ell} \rightarrow 0$ and $m_{\ell} \rightarrow \infty$, (1) becomes the wellknown Lognormal pdf. The cdf and the $n$ th-order moment of $R_{\ell}$ can be expressed as

$$
F_{R_{\ell}}(r)=1-\frac{1}{\Gamma\left(m_{\ell}\right)} \Gamma\left(m_{\ell}, \frac{m_{\ell}}{\Omega_{\ell}} r^{\beta_{\ell}}\right)
$$

and

$$
\mathcal{E}\left\langle R_{\ell}^{n}\right\rangle=\left(\frac{\Omega_{\ell}}{m_{\ell}}\right)^{n / \beta_{\ell}} \frac{\Gamma\left(m_{\ell}+n / \beta_{\ell}\right)}{\Gamma\left(m_{\ell}\right)}
$$

\footnotetext{
${ }^{2}$ As pointed out in [4], the pdf of (1), introduced by Stacy in [1], is different from the type of generalization for the Gamma distribution presented in [17] which models the power of Rice-faded envelopes.
}

respectively, where $n$ is a positive number and $\Gamma(\cdot, \cdot)$ is the upper incomplete Gamma function [16, eq. (8.350/2)].

\section{A. Distribution of the Product of $G G$ Variates}

Let us define another RV, $Y$, as the product of the $N$ GG distributed RVs $R_{\ell}$, i.e.,

$$
Y \triangleq \prod_{i=1}^{N} R_{i}
$$

with $\beta_{\ell}$ 's belong to rationals.

Theorem 1 (Moments-generating function): The momentsgenerating function (mgf) of $Y$ is given by

$$
\mathcal{M}_{Y}(s)=V G_{p, n}^{n, p}\left[\begin{array}{l|c}
W s^{n} & \left.\begin{array}{c}
I_{n}\left(\beta_{\ell} ; 1-m_{\ell}\right) \\
\Delta(n ; 0)
\end{array}\right]
\end{array}\right.
$$

where $G[\cdot]$ is the Meijer's G-function ${ }^{3}$ [16, eq. (9.301)], $I_{n}\left(\beta_{\ell} ; 1-m_{\ell}\right) \triangleq \Delta\left(n / \beta_{1} ; 1-m_{1}\right), \quad \Delta\left(n / \beta_{2} ; 1-\right.$ $\left.m_{2}\right), \ldots, \Delta\left(n / \beta_{N} ; 1-m_{N}\right)$, and $\Delta(k ; x)$ is defined as $\Delta(k ; x) \triangleq x / k,(x+1) / k, \ldots,(x+k-1) / k$, with $x$ being an arbitrary real value and $k$ a positive integer,

$$
V=\sqrt{n}(\sqrt{2 \pi})^{N+1-n-p} \prod_{i=1}^{N} \frac{\left(n / \beta_{i}\right)^{m_{i}-1 / 2}}{\Gamma\left(m_{i}\right)}
$$

and

$$
W=\frac{1}{n^{n}} \prod_{i=1}^{N}\left(\frac{n \Omega_{i}}{m_{i} \beta_{i}}\right)^{n / \beta_{i}} .
$$

Moreover, $n$ and $p$ are two positive integers defined as

$$
n \triangleq \prod_{i=1}^{N} k_{i}
$$

and

$$
p \triangleq n \sum_{i=1}^{N} \frac{1}{\beta_{i}}
$$

under the constraint that

$$
l_{\ell}=\frac{1}{\beta_{\ell}} \prod_{i=1}^{\ell} k_{i}
$$

is a positive integer, with $k_{\ell}$ and $l_{\ell}$ being also positive integers.

Proof: The proof is given in the Appendix.

The values for $k_{\ell}$ and $l_{\ell}$ can be found as follows: Depending upon the value $\beta_{1}$ taken in (6e), $k_{1}$ and $l_{1}$ are two minimum positive integers such that $l_{1}=k_{1} / \beta_{1}$ holds (e.g. for $\beta_{1}=3.6$, $k_{1}=18$ and $l_{1}=5$ ). Similarly, $k_{\ell}$ and $l_{\ell}$ are two minimum positive integers such that (6e) holds. Note that for identical and integer order fading parameters (5) significantly simplifies.

\footnotetext{
${ }^{3}$ Note that $G[\cdot]$ can be expressed in terms of more familiar generalized hypergeometric functions ${ }_{p} F_{q}(\cdot ; \cdot ; \cdot)[16$, eq. $(9.14 / 1)]$ using the transformation presented in [16, eq. (9.303)], with $p$ and $q$ being positive integers. In addition, both $G[\cdot]$ and ${ }_{p} F_{q}(\cdot ; \cdot ; \cdot)$ are included as built-in functions in most of the popular mathematical software packages such as Maple ${ }^{\mathrm{TM}}$ or Mathematica ${ }^{\mathrm{TM}}$.
} 
Thus, for $\beta_{\ell}=\beta \forall \ell$, with $\beta \in \mathcal{N}$ being integer, then $n=\beta$, $p=N$, and $l_{\ell}=1$, and hence, (5) reduces to

$$
\begin{aligned}
& \mathcal{M}_{Y}(s)=\frac{\sqrt{\beta}(\sqrt{2 \pi})^{1-\beta}}{\prod_{i=1}^{N} \Gamma\left(m_{i}\right)} \\
& \times G_{N, \beta}^{\beta, N}\left[\left.\left(\frac{s}{\beta}\right)^{\beta} \prod_{i=1}^{N} \frac{\Omega_{i}}{m_{i}}\right|_{\Delta(\beta ; 0)} ^{1-m_{1}, 1-m_{2}, \ldots, 1-m_{N}}\right]
\end{aligned}
$$

which for $m_{\ell}=1$ and $N=1$ reduces to a known result [7, eq. (5)].

Lemma 1 (Probability density function): The pdf of $Y$ is given by

$$
f_{Y}(y)=\frac{\sqrt{n} V(\sqrt{2 \pi})^{n-1}}{y} G_{p, 0}^{0, p}\left[\frac{W n^{n}}{y^{n}} \mid \begin{array}{c}
I_{n}\left(\beta_{\ell} ; 1-m_{\ell}\right) \\
-
\end{array}\right] .
$$

Proof: Applying the inverse Laplace transform $\mathcal{L}^{-1}(\cdot ; \cdot)$ [16, Sec. 17.11] in (5), the pdf of $Y$ [16, Sec. 17.11]

$$
f_{Y}(y)=\mathcal{L}^{-1}\left\{\mathcal{M}_{Y}(s) ; y\right\}
$$

can be obtained in closed form using [18, eq. (21)] as in (8).

Lemma 2 (Cumulative distribution function): The cdf of $Y$ is given by

$$
F_{Y}(y)=\frac{V(\sqrt{2 \pi})^{n-1}}{\sqrt{n}} G_{1, p+1}^{p, 1}\left[\left.\frac{y^{n}}{W n^{n}}\right|_{I_{n}\left(\beta_{\ell} ; m_{\ell}\right), 0} ^{1}\right] .
$$

Proof: Since the cdf of $Y$ is given by

$$
F_{Y}(y)=\int_{0}^{y} f_{Y}(x) d x
$$

by using (8) and [18, eq. (26)], (10) can be easily obtained.

Note that for $m_{\ell}=1$ and $\beta_{\ell}=2 \forall \ell$, (10) can be reduced to $[15$, eq. (19)].

\section{B. Distribution of the Sum of $G G$ Variates}

Let us define $S$ to be the sum of $N$ GG RVs, i.e.,

$$
S \triangleq \sum_{i=1}^{N} R_{i}
$$

Theorem 2 (A cdf bound of the sum of $G G R V s$ ): The cdf of $S$ is upper bounded as

$$
F_{S}(y) \leq F_{Y}\left[\left(\frac{y}{N}\right)^{N}\right]
$$

Proof: Using the well-known inequality for the arithmetic and geometric means [16, Sec. 11.116]

$$
\mathcal{A}_{N} \geq \mathcal{G}_{N}
$$

with

$$
\mathcal{A}_{N} \triangleq \frac{1}{N} \sum_{i=1}^{N} R_{i}
$$

and

$$
\mathcal{G}_{N} \triangleq \prod_{i=1}^{N} R_{i}^{1 / N}
$$

being the arithmetic and geometric means, respectively, $S$ can be lower bounded as

$$
S \geq N \prod_{i=1}^{N} R_{i}^{1 / N}
$$

Using (4), (10), and (17), it can be easily seen that the cdf of $S$ can be upper bounded as in (13).

It is interesting to note that the problem of obtaining an upper bound for the cdf of $S$ with nonidentically distributed RVs ( $\Omega_{\ell}$ : average powers) may be equivalently stated as finding an upper bound for the cdf of a weighted sum of $N$ i.i.d. RVs having equal average powers each, $\Omega$, with weights $w_{\ell}=\sqrt{\Omega_{\ell} / \Omega}$.

\section{PERformance AnAlysis of EQUAL-GAin DIVERSITY RECEIVERS}

Let us consider an $N$-branch EGC receiver operating over independent, but not necessarily identically distributed, GG fading channels. The baseband received signal in the $\ell$ th $(\ell=1,2, \ldots, N)$ antenna is $\zeta_{\ell}=w R_{\ell} \exp \left(\jmath \psi_{\ell}\right)+n_{\ell}$, where $w$ is the complex transmitted symbol, with $E_{s}=$ $\mathcal{E}\left\langle|w|^{2}\right\rangle$ being the transmitted average symbols' energy, $R_{\ell}$ is the instantaneous fading envelope being modeled as a GG distributed $\mathrm{RV}, \psi_{\ell}$ is the instantaneous phase of the channel, and $n_{\ell}$ is the instantaneous additive white Gaussian noise (AWGN) sample with single-sided power spectral density $N_{0}$ identical for all channels. The usual assumption is made that the $\psi_{\ell}$ 's are known to the receiver.

The instantaneous SNR per symbol of the $\ell$ th diversity channel can be expressed as

$$
\gamma_{\ell}=R_{\ell}^{2} \frac{E_{s}}{N_{0}}
$$

with its corresponding average SNR being

$$
\bar{\gamma}_{\ell}=\mathcal{E}\left\langle R_{\ell}^{2}\right\rangle \frac{E_{s}}{N_{0}}=\left(m_{\ell}\right)_{2 / \beta_{\ell}}\left(\frac{\Omega_{\ell}}{m_{\ell}}\right)^{2 / \beta_{\ell}} \frac{E_{s}}{N_{0}}
$$

where $(\xi)_{u}$ is the Pochhammer symbol defined as $(\xi)_{u}=$ $\Gamma(\xi+u) / \Gamma(\xi)$. Based on an interesting property of the GG distribution, that the $n$th power of a GG distributed RV with parameters $\left(m_{\ell}, \beta_{\ell}, \Omega_{\ell}\right)$ is another GG distributed RV with parameters $\left(m_{\ell}, \beta_{\ell} / n, \Omega_{\ell}\right)$, it can be easily concluded that $\gamma_{\ell}$ is also a GG distributed RV with parameters $\left(m_{\ell}, \beta_{\ell} / 2,\left(\Xi_{\ell} \bar{\gamma}_{\ell}\right)^{\beta_{\ell} / 2}\right)$ where $\Xi_{\ell}=1 /\left(m_{\ell}\right)_{2 / \beta_{\ell}}$. Hence, by using the formulae for $\left\{R_{\ell}\right\}$ given by (1)-(3), the corresponding expressions for $\left\{\gamma_{\ell}\right\}$ can be easily derived, replacing $\beta_{\ell}$ with $\beta_{\ell} / 2$ and $\Omega_{\ell} / m_{\ell}$ with $\left(\Xi_{\ell} \bar{\gamma}_{\ell}\right)^{\beta_{\ell} / 2}$ helping us to study the performance of multi-branch diversity receivers operating over GG fading channels. For example, from (2), the cdf of $\gamma_{\ell}$ can be derived as

$$
F_{\gamma_{\ell}}(\gamma)=1-\frac{1}{\Gamma\left(m_{\ell}\right)} \Gamma\left[m_{\ell},\left(\frac{\gamma}{\Xi_{\ell} \bar{\gamma}_{\ell}}\right)^{\beta_{\ell} / 2}\right] \text {. }
$$

According to the above mentioned property, hereafter in this paper, $V$ and $W$ are modified as

$$
V=\sqrt{n}(\sqrt{2 \pi})^{N+1-n-p} \prod_{i=1}^{N} \frac{\left(2 n / \beta_{i}\right)^{m_{i}-1 / 2}}{\Gamma\left(m_{i}\right)}
$$


and

$$
W=\frac{1}{n^{n}} \prod_{i=1}^{N}\left(\Xi_{i} \bar{\gamma}_{i}\right)^{n}\left(\frac{2 n}{\beta_{i}}\right)^{2 n / \beta_{i}}
$$

with $p=2 n \sum_{i=1}^{N}\left(1 / \beta_{i}\right)$ and $l_{\ell}=2\left(\prod_{i=1}^{\ell} k_{i}\right) / \beta_{\ell}$.

\section{A. Moments of the Output SNR}

The instantaneous EGC output SNR per symbol is given by

$$
\gamma_{\mathrm{egc}}=\frac{1}{N}\left(\sum_{i=1}^{N} \sqrt{\gamma_{i}}\right)^{2}
$$

Using the multinomial identity [19, eq. (24.1.2)], the $n$ th-order moment of $\gamma_{\text {egc }}, \mu_{n}=\mathcal{E}\left\langle\gamma_{\text {egc }}^{n}\right\rangle$, can be derived as

$$
\begin{aligned}
\mu_{n} & =\frac{1}{N^{n}} \mathcal{E}\left\langle\left(\sum_{i=1}^{N} \sqrt{\gamma_{i}}\right)^{2}\right\rangle \\
& =\frac{(2 n) !}{N^{n}} \sum_{\substack{k_{1}, k_{2}, \ldots, k_{N}=0 \\
k_{1}+k_{2}+\cdots+k_{N}=2 n}}^{2 n} \mathcal{E}\left\langle\prod_{i=1}^{N} \gamma_{i}^{k_{i} / 2} / k_{i} !\right\rangle .
\end{aligned}
$$

Since the diversity input branches are uncorrelated, the mean product term in the above equation can be expressed as

$$
\mathcal{E}\left\langle\prod_{i=1}^{N} \gamma_{i}^{k_{i} / 2}\right\rangle=\prod_{i=1}^{N} \mathcal{E}\left\langle\gamma_{i}^{k_{i} / 2}\right\rangle
$$

By substituting (3) and (24) in (23), the moments of the EGC output SNR for independent but not necessarily identically distributed input branches can be expressed in closed form as

$$
\mu_{n}=\frac{(2 n) !}{N^{n}} \sum_{\substack{k_{1}, k_{2}, \ldots, k_{N}=0 \\ k_{1}+k_{2}+\cdots+k_{N}=2 n}}^{2 n} \prod_{i=1}^{N} \frac{\left(\Xi_{i} \bar{\gamma}_{i}\right)^{k_{i} / 2}}{k_{i} ! \Gamma\left(m_{i}\right)} \Gamma\left(m_{i}+\frac{k_{i}}{\beta_{i}}\right) \text {. }
$$

1) Average output SNR: By setting $n=1$ in (25), the EGC average output SNR per symbol, $\bar{\gamma}_{\text {egc }}=\mu_{1}$, can be obtained in closed form as

$$
\begin{aligned}
\bar{\gamma}_{\text {egc }}=\frac{1}{N}\left[\sum_{i=1}^{N} \bar{\gamma}_{i}+2\right. & \sum_{i=2}^{N} \sum_{j=1}^{i-i} \frac{\sqrt{\Xi_{i} \Xi_{j} \bar{\gamma}_{i} \bar{\gamma}_{j}}}{\Gamma\left(m_{i}\right) \Gamma\left(m_{j}\right)} \\
& \left.\times \Gamma\left(m_{i}+\frac{1}{\beta_{i}}\right) \Gamma\left(m_{j}+\frac{1}{\beta_{j}}\right)\right] .
\end{aligned}
$$

For $m_{\ell}=1$ and $\beta_{\ell}=\beta \forall \ell$, (26) reduces to a known expression [8, eq. (18)] for Weibull fading channels. Moreover, for $m_{\ell}=m$ and $\beta_{\ell}=2 \forall \ell$, (26) reduces to another known expression [20, eq. (19)] for Nakagami- $m$ fading channels.

2) Amount of fading: By using (25), the amount of fading (AoF), defined as the ratio of the variance to the square average SNR per symbol, i.e., $A_{F} \triangleq \operatorname{var}\left(\gamma_{\text {egc }}\right) / \bar{\gamma}_{\text {egc }}^{2}$, can be expressed in a simple closed form as

$$
A_{F} \triangleq \frac{\mu_{2}}{\mu_{1}^{2}}-1
$$

\section{B. Outage Probability}

By using (12), (17), (18), and (22), a lower bound for $\gamma_{\mathrm{egc}}$ can be expressed as $\gamma_{\text {egc }} \geq \gamma_{\text {egc }}^{*}=N \prod_{i=1}^{N} \gamma_{i}^{1 / N}$. The cdf of $\gamma_{\text {egc }}^{*}$ can be derived by substituting $y=(\gamma / N)^{N}$ in (10), i.e., $F_{\gamma_{\mathrm{egc}}^{*}}(\gamma)=\frac{V(\sqrt{2 \pi})^{n-1}}{\sqrt{n}} G_{1, p+1}^{p, 1}\left[\left.\frac{(\gamma / N)^{n N}}{W n^{n}}\right|_{I_{2 n}\left(\beta_{\ell} ; m_{\ell}\right), 0} ^{1}\right]$.

If $\gamma_{\text {th }}$ is a certain specified threshold, then the outage probability is defined as the probability that $\gamma_{\text {egc }}$ falls below $\gamma_{\text {th }}$. An upper bound for this probability can be obtained by replacing $\gamma$ with $\gamma_{\text {th }}$ in (28) as

$$
P_{\text {out }}\left(\gamma_{\text {th }}\right) \leq F_{\gamma_{\text {egc }}^{*}}\left(\gamma_{\text {th }}\right)
$$

\section{Average Bit Error Probability}

One straightforward approach to obtain a bound for the ABEP, $\bar{P}_{\text {be }}$, is to average the conditional bit error probability, $P_{\mathrm{be}}(\gamma)$, over the pdf of $\gamma_{\text {egc }}^{*}$, i.e.,

$$
\bar{P}_{\text {be }} \leq \int_{0}^{\infty} P_{\text {be }}(\gamma) f_{\gamma_{\text {egc }}^{*}}(\gamma) d \gamma
$$

By taking the first derivative of $F_{\gamma_{\text {egc }}^{*}}(\gamma)$ in (28) with respect to $\gamma$, the corresponding pdf can be obtained as

$$
f_{\gamma_{\mathrm{egc}}^{*}}(\gamma)=\frac{N \sqrt{n} V}{\gamma(\sqrt{2 \pi})^{1-n}} G_{0, p}^{p, 0}\left[\left.\frac{(\gamma / N)^{n N}}{W n^{n}}\right|_{I_{2 n}\left(\beta_{\ell} ; m_{\ell}\right)} ^{-}\right]
$$

Moreover, for $P_{\mathrm{be}}(\gamma)$ there are well-known generic expressions for two different sets of modulation schemes:

i) BPSK, BFSK, $M$-ary differentially encoded phaseshift keying ( $M$-DEPSK), quadrature phase-shift keying (QPSK), $M$-ary phase-shift keying ( $M$-PSK), $M$-ary frequency-shift keying ( $M$-FSK), and $M$-ary differential PSK ( $M$-DPSK) in the form of

$$
P_{\mathrm{be}}(\gamma)=A \operatorname{erfc}(\sqrt{B \gamma})
$$

where $\operatorname{erfc}(\cdot)$ is the well-known complementary error function [16, eq. (8.250/4)];

ii) Differential BPSK (DBPSK) and $M$-ary noncoherent FSK ( $M$-NFSK), in the form of

$$
P_{\mathrm{be}}(\gamma)=A \exp (-B \gamma)
$$

The particular values of $A$ and $B$ in (32) and (33) depend on the specific modulation scheme employed and can be found in [21]. Next, (30) is solved in closed form for each of the above two sets of signals.

1) $M$-PSK, $M$-FSK, $M$-DEPSK, and $M$-DPSK: Using (30), (31), and (32), it can be easily recognized that for this first set of modulation schemes, the evaluation of definite integrals, which include Meijer's, power, and exponential functions, is required. Since such integrals are not tabulated, the solution can be found with the aid of [18, eq. (21)], and thus the ABEP can be upper bounded as

$$
\begin{aligned}
& \bar{P}_{\text {be }} \leq \frac{A V}{\sqrt{\pi n}} \frac{1}{(\sqrt{2 \pi})^{n(N-1)}} \\
& \times G_{2 n N, p+n N}^{p \underset{2 n N}{2 n}}\left[\frac{n^{n(N-1)}}{W B^{n N}} \mid \begin{array}{c}
\Delta(n N ; 1), \Delta(n N ; 1 / 2) \\
I_{2 n}\left(\beta_{\ell} ; m_{\ell}\right), \Delta(n N ; 0)
\end{array}\right] .
\end{aligned}
$$




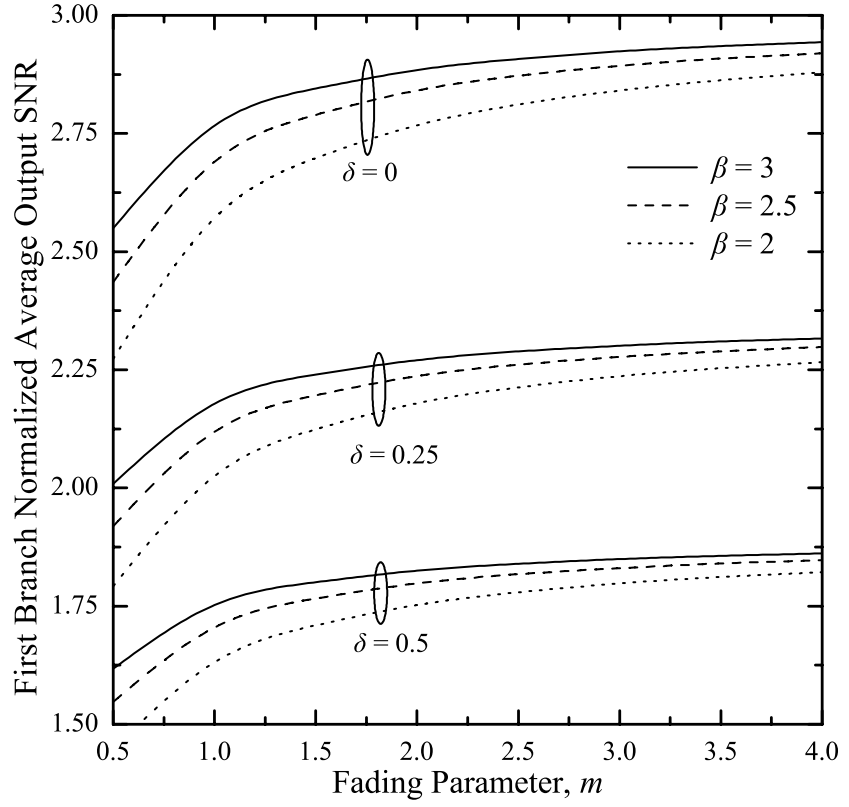

Fig. 1. First branch normalized EGC average output SNR as a function of $m$ for $N=3$.

2) DBPSK and M-NFSK: Similarly to the first set, for this second set (i.e., for DBPSK and $M$-NFSK), an upper bound for the ABEP can be derived as

$$
\bar{P}_{\text {be }} \leq \frac{A V \sqrt{N}}{(\sqrt{2 \pi})^{n(N-1)}} G_{n N, p}^{p, n N}\left[\left.\frac{n^{n(N-1)}}{W B^{n N}}\right|_{I_{2 n}\left(\beta_{\ell} ; m_{\ell}\right)} ^{\Delta(n N ; 1)}\right] .
$$

\section{Performance Evaluation and Discussion}

In this section, using the previous mathematical analysis, numerical and simulation results are presented for the performance of EGC receivers operating over GG fading channels. For these performance evaluation results we consider the general case of not necessarily equal $\bar{\gamma}_{\ell}$ 's. Particularly, an exponentially decaying power delay profile (PDP) is adopted

$$
\bar{\gamma}_{\ell}=\bar{\gamma}_{1} \exp [-\delta(\ell-1)]
$$

with $\delta$ being the power decaying factor. For the convenience of the presentation of the performance evaluation results and without any loss of generality, it will be assumed that $m_{\ell}=m$ and $\beta_{\ell}=\beta \forall \ell$.

Using (26), Fig. 1 presents the first branch normalized average output SNR, $\gamma_{\text {egc }} / \bar{\gamma}_{1}$, of a three-branch EGC receiver as a function of $m$, for several values of $\beta$ and $\delta$. It is noted that as $m$ and/or $\beta$ increases, $\gamma_{\text {egc }} / \bar{\gamma}_{1}$ improves, while the combining loss of the receiver gets more accentuated as $\delta$ increases. Similar behavior has been also observed in [20] for Nakagami- $m$ fading channels. By numerically evaluating (27), in Fig. 2, $A_{F}$ is plotted as a function of $\beta$, for $N=2$ and for several values of $m$. As expected, $A_{F}$ decreases as $\beta$ and/or $m$ increases.

Having numerically evaluated (29), in Fig. 3, upper bounds for $P_{\text {out }}$ are presented as a function of the normalized outage threshold, $\gamma_{\text {th }} / \bar{\gamma}$, for $m=2$, i.i.d. input branches (i.e., $\bar{\gamma}_{\ell}=\bar{\gamma} \forall \ell$ ), and different values of $\beta$ and $N$. The obtained results clearly show that $P_{\text {out }}$ improves with an increase of $N$

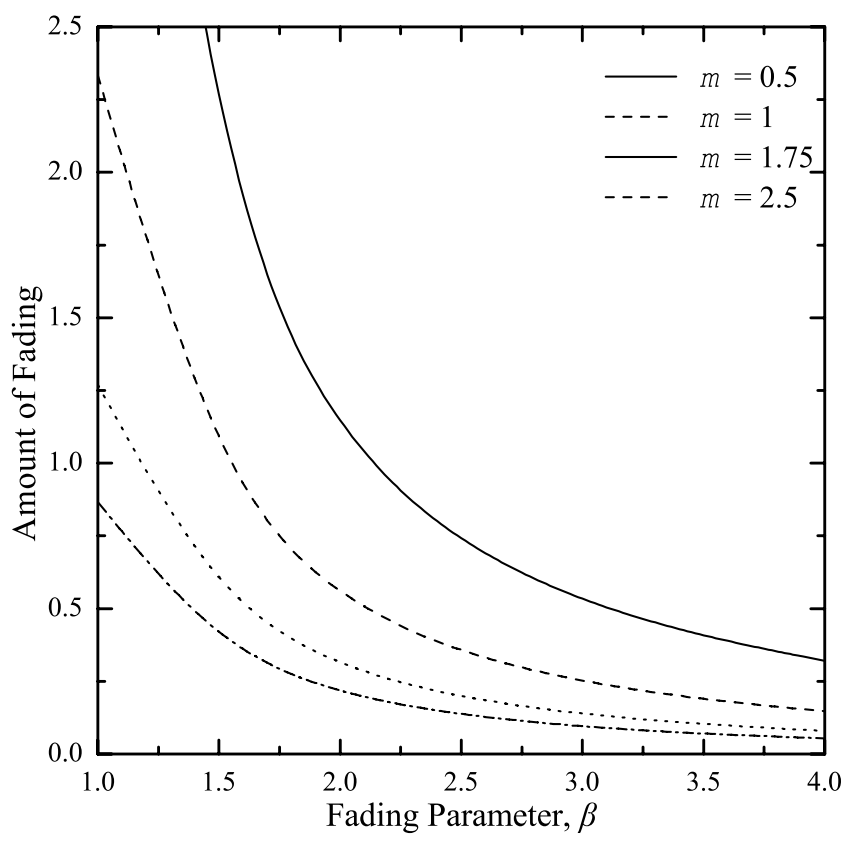

Fig. 2. Amount of fading at the output of the combiner as a function of $\beta$ for $N=2$ and i.i.d. branch SNRs.

and/or $\beta$. In order to verify the tightness of the bounds, curves obtained by means of computer simulations are also included for comparison purposes. By comparing the performances it is evident that the numerical results for the bounds (see (29)) are very close to the equivalent simulated ones which represent the exact $P_{\text {out }}$ performance. This observation clearly demonstrates the accuracy of the proposed bounds. It is also noted that as $\beta$ increases, the proposed bounds become even tighter. However, as $N$ increases, the difference between the two performance results slightly increases. In Fig. $4, P_{\text {out }}$ is plotted as a function of the first branch normalized outage threshold, $\gamma_{\mathrm{th}} / \bar{\gamma}_{1}$, for $\beta=2.5, N=2$, and $\bar{\gamma}_{1}=0.5 \bar{\gamma}_{2}$. These results suggest that the higher $m$ is, the smaller are the differences between numerical and computer simulation results for $P_{\text {out }}$. For example, at $P_{\text {out }}=10^{-3}$, the differences between them for $m=1,2$, and 4 are less than 2, 1 , and 0.5 $\mathrm{dB}$, respectively. The trend of the performance, as illustrated in Figs. 3 and 4, can be explained as follows. It is clear that the smaller the difference between the terms of the left hand side (LHS) and right hand side (RHS) of (14), the tighter are the bounds. In fact, the equality in (14) holds if and only if all $R_{\ell}$ 's are equal with each other, i.e., $R_{1}=R_{2}=\cdots=R_{N}$. For relatively large values of $m_{\ell}$ 's and/or $\beta_{\ell}$ 's, all fading envelopes $R_{\ell}$ will be, with high probability, close to their average value, and thus, it is expected that $R_{\ell}$ 's will take similar values. As for $N$, the smaller its value is the tighter are the bounds. This happens because both bounds and exact curves move towards the performance obtained for $N=1$. In fact, from (14) it can be seen that for $N=1$ these two curves coincide.

Using (34) and (35), the ABEP performance of an $N$-branch EGC receiver for several coherent and noncoherent binary and multilevel modulation schemes has been obtained. In Figs. 5 and 6 , the ABEP, $\bar{P}_{\mathrm{be}}$, of DBPSK is plotted as a function of $\bar{\gamma}$ for i.i.d. fading statistics. In Fig. $5, \bar{P}_{\text {be }}$ is plotted as a function of $\bar{\gamma}$, for $\beta=3$ and several values of $m$. As expected, 


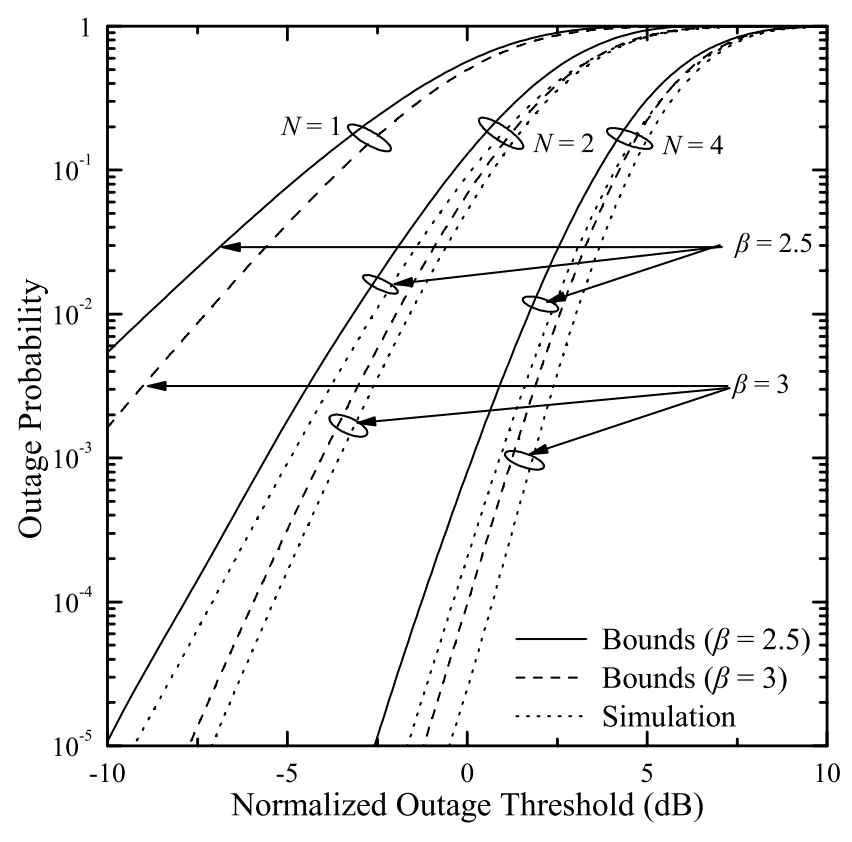

Fig. 3. Outage probability as a function of the average input SNR for $m=2$ and i.i.d. branch SNRs.

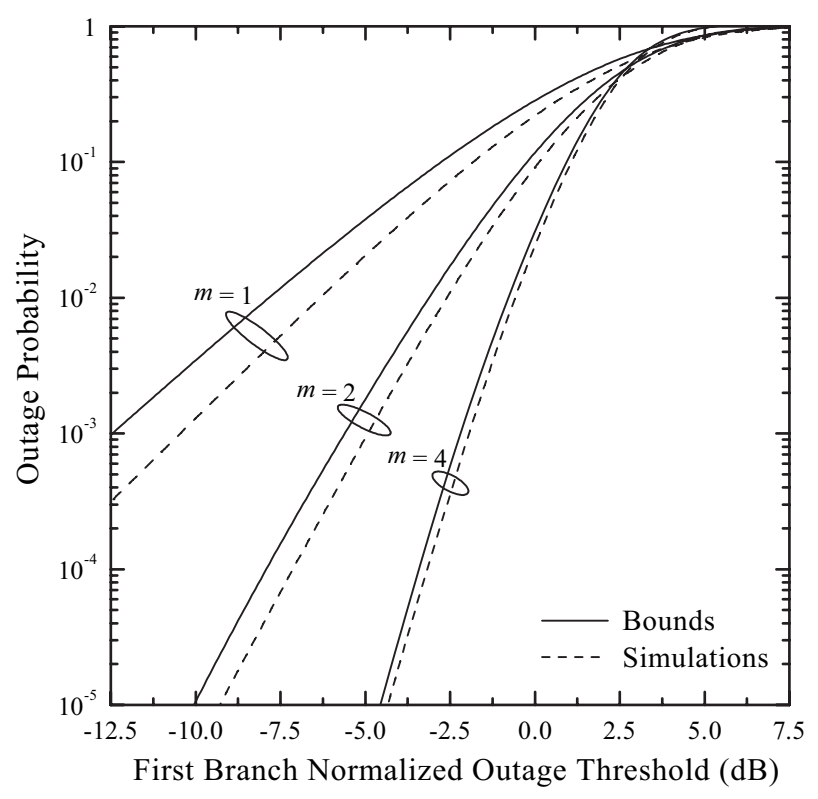

Fig. 4. Outage probability as a function of the first branch normalized average input SNR for $\beta=2.5$ and $\bar{\gamma}_{1}=0.5 \bar{\gamma}_{2}$.

the obtained performance evaluation results show that $\bar{P}_{\text {be }}$ improves with an increase of $\bar{\gamma}$. For comparison purposes the curves for the corresponding exact $\bar{P}_{\text {be }}$, obtained via computer simulations, are also included in the same figure. By comparing the numerically evaluated results with the computer simulated ones, we deduce a close match between them. For example in Fig. 5, for $\bar{P}_{\text {be }}=10^{-5}, N=6$ and $m=2$, their difference is less than $0.5 \mathrm{~dB}$. In Fig. $6, \bar{P}_{\text {be }}$ is plotted as a function of $\bar{\gamma}$, for $m=2$ and several values of $\beta$. As for $m, \beta$, and $N$, similar findings with those observed from Figs. 3 and 4 can also be extracted. Fig. 7 presents $\bar{P}_{\text {be }}$ of $M$-PSK with

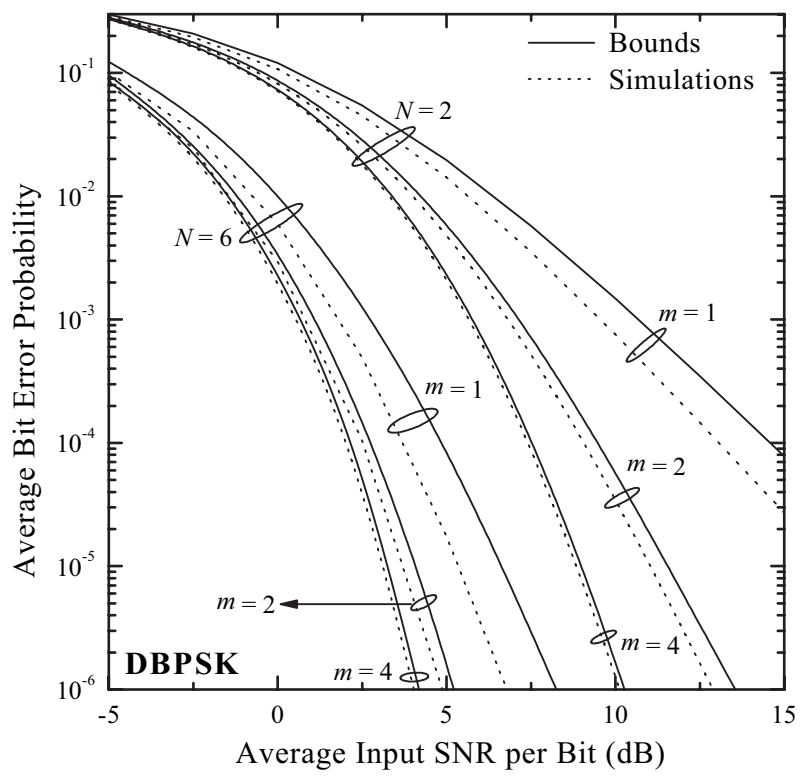

Fig. 5. ABEP of EGC with DBPSK modulation format as a function of the average input SNR per bit for $\beta=3$ and i.i.d. branch SNRs.

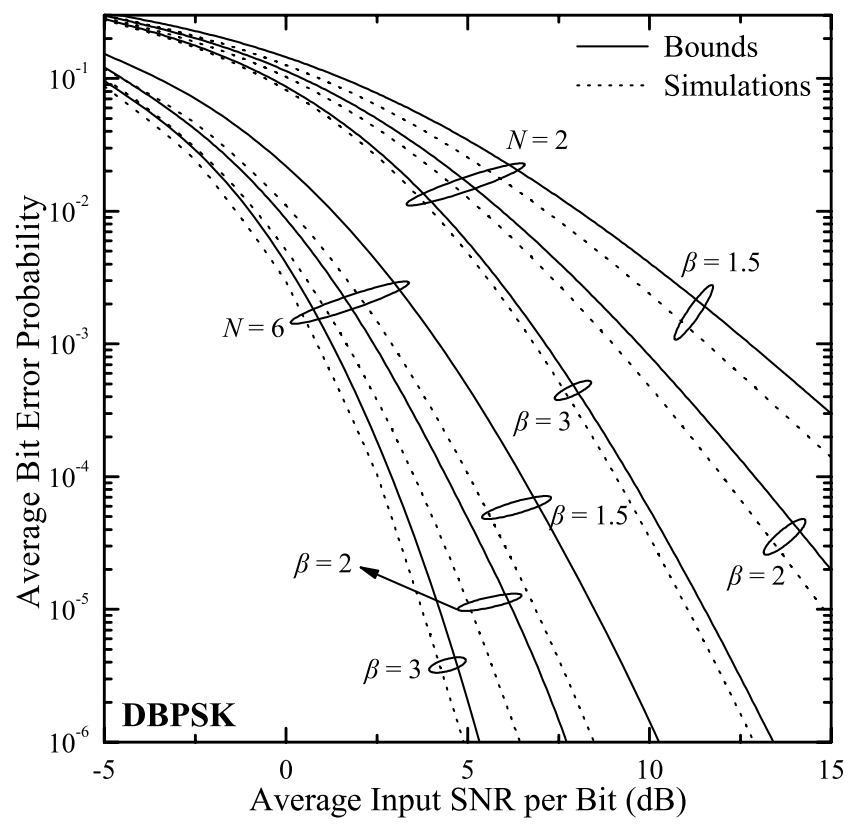

Fig. 6. ABEP of EGC with DBPSK modulation format as a function of the average input SNR per bit for $m=2$ and i.i.d. branch SNRs.

Gray encoding as a function of $\bar{\gamma}$ for $N=3, m=2, \beta=2.5$, i.i.d. input branches, and several values of $M$. As expected, for a fixed $\bar{\gamma}, \bar{P}_{\text {be }}$ degrades with increasing $M$. Furthermore, the higher $M$, the tighter the bounds. Finally, in Fig. $8, \bar{P}_{\text {be }}$ of 8-PSK with Gray encoding is plotted as a function of $\bar{\gamma}_{1}$ for $\beta=2.5, \delta=0.2$, and $M=8$. Again here we note the tightness of the proposed bounds.

\section{CONCLUSIONS}

Capitalizing on the product of $N$ GG RVs, its mgf, pdf, and cdf were obtained in closed form. These expressions were used to derive a closed-form union upper bound for the cdf 


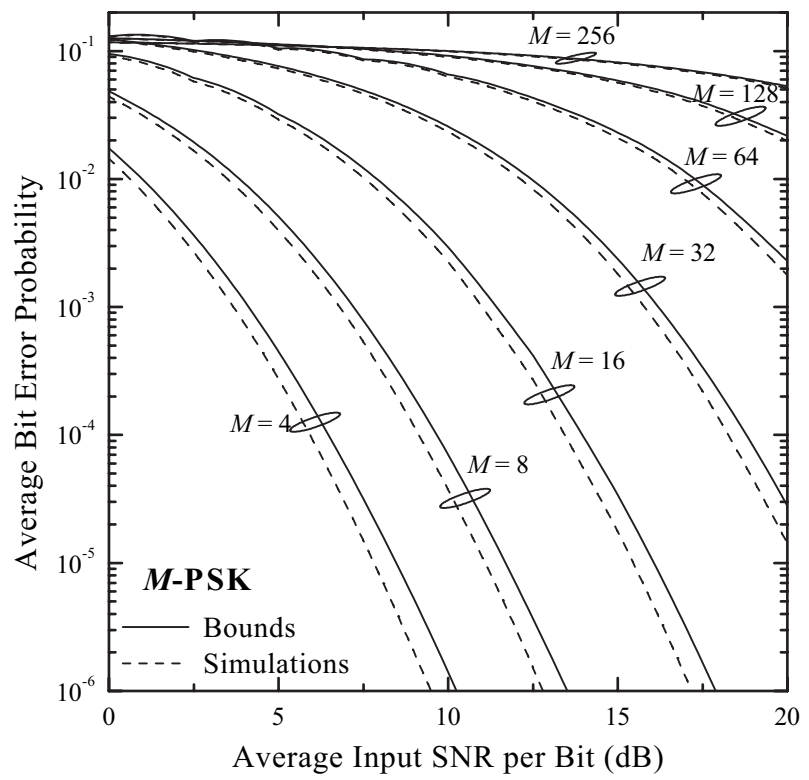

Fig. 7. ABEP of a triple-branch EGC with Gray encoded $M$-PSK modulation as a function of the average input SNR per bit for $m=2, \beta=2.5$, and i.i.d. branch SNRs.

of the sum of GG distributed RVs. Based on this bound, the performance of $N$-branch EGC receivers operating over GG fading channels was studied and important performance measures such as the outage probability and the ABEP were obtained in closed form. By comparing numerical evaluation and computer simulation results, it was shown that the higher the value of $m$ and/or $\beta$ and/or the smaller the value of $N$, the tighter are the proposed bounds.

\section{ACKNOWLEDGMENT}

This work has been performed within the framework of the Satellite Network of Excellence (SatNEx) project, a Network of Excellence (NoE) funded by European Committee (EC) under the FP6 program.

\section{APPENDIX \\ PROOF OF THEOREM 1}

In order to prove Theorem 1, we follow a similar procedure as in [22]. Starting from the definition of the mgf of $Y$, i.e.,

$$
\mathcal{M}_{Y}(s)=\mathcal{E}\langle\exp (-s Y)\rangle
$$

and by using (1) and (4), $\mathcal{M}_{Y}(s)$ can be written as

$$
\begin{aligned}
\mathcal{M}_{Y}(s)=\left[\prod_{i=1}^{N}\left(\frac{m_{i}}{\Omega_{i}}\right)^{m_{i}} \frac{\beta_{i}}{\Gamma\left(m_{i}\right)}\right] \\
\times \int_{0}^{\infty} \cdots \int_{0}^{\infty} \int_{0}^{\infty}\left(\prod_{i=1}^{N} r_{i}^{m_{i} \beta_{i}-1}\right) \exp \left(-s \prod_{i=1}^{N} r_{i}\right) \\
\quad \times \exp \left(-\sum_{i=1}^{N} \frac{m_{i}}{\Omega_{i}} r_{i}^{\beta_{i}}\right) d r_{1} d r_{2} \cdots d r_{N}
\end{aligned}
$$

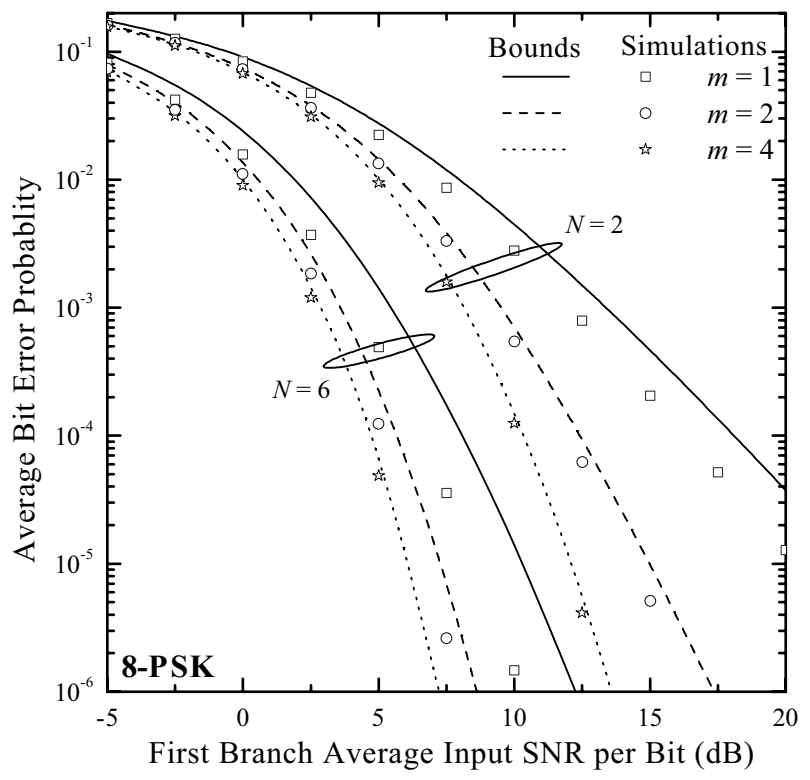

Fig. 8. ABEP of EGC with Gray encoded 8-PSK modulation as a function of the average SNR per bit of the first input branch for $\beta=2.5$ and $\delta=0.2$.

which after applying the transformation $z_{\ell}=r_{\ell}^{\beta_{\ell}}$ can be rewritten as

$$
\begin{aligned}
\mathcal{M}_{Y}(s)=\left[\prod_{i=1}^{N}\left(\frac{m_{i}}{\Omega_{i}}\right)^{m_{i}} \frac{1}{\Gamma\left(m_{i}\right)}\right] \\
\times \int_{0}^{\infty} \cdots \int_{0}^{\infty} \int_{0}^{\infty}\left(\prod_{i=1}^{N} z_{i}^{m_{i}-1}\right) \exp \left(-s \prod_{i=1}^{N} z_{i}^{1 / \beta_{i}}\right) \\
\quad \times \exp \left(-\sum_{i=1}^{N} \frac{m_{i}}{\Omega_{i}} z_{i}\right) d z_{1} d z_{2} \cdots d z_{N}
\end{aligned}
$$

With the aid of [18, eq. (11)], the above multiple integrals can be expressed in terms of Meijer's functions as

$$
\begin{aligned}
& \mathcal{M}_{Y}(s)=\left[\prod_{i=1}^{N}\left(\frac{m_{i}}{\Omega_{i}}\right)^{m_{i}} \frac{1}{\Gamma\left(m_{i}\right)}\right] \\
& \times \int_{0}^{\infty} z_{N}^{m_{N}-1} G_{0,1}^{1,0}\left[\left.\frac{m_{N}}{\Omega_{N}} z_{N}\right|_{0} ^{-}\right] \cdots \int_{0}^{\infty} z_{2}^{m_{2}-1} \\
& \times G_{0,1}^{1,0}\left[\frac{m_{2}}{\Omega_{2}} z_{2} \mid \begin{array}{c}
- \\
0
\end{array}\right] \int_{0}^{\infty} z_{1}^{m_{1}-1} G_{0,1}^{1,0}\left[\left.s \prod_{i=1}^{N} z_{i}^{1 / \beta_{i}}\right|_{0} ^{-}\right] \\
& \times G_{0,1}^{1,0}\left[\left.\frac{m_{1}}{\Omega_{1}} z_{1}\right|_{0} ^{-}\right] d z_{1} d z_{2} \cdots d z_{N} .
\end{aligned}
$$

In order to solve the above $N$-fold integral, the following properties for $\Delta(\cdot ; \cdot)$ should be taken into consideration:

Property 1: For any real $x$ and positive integers $k$ and $p$, it holds that

$$
\Delta[k ; \Delta(p ; x)]=\Delta(k p ; x) .
$$

Property 2: For any real $x$ and integer $k$, it holds that the sum of terms included in $\Delta(k ; x)$, i.e., $\mathcal{S}=\sum_{i=0}^{N-1}(x+i) / k$, is given by

$$
\mathcal{S}=x+\frac{k-1}{2} .
$$


Starting from the inner integral (i.e., that on $z_{1}$ ), continuing towards the outer one (i.e., that on $z_{N}$ ), and using Properties 1 and 2 , successive integrations of the form

$$
\int_{0}^{\infty} z_{\ell}^{m_{\ell}-1} G_{c, d}^{a, b}\left[\Psi z_{\ell}^{\varphi} \mid \begin{array}{c}
H \\
J
\end{array}\right] G_{0,1}^{1,0}\left[\left.\frac{m_{\ell}}{\Omega_{\ell}} z_{\ell}\right|_{0} ^{-}\right] d z_{\ell}
$$

arise due to the fact that each of these integrals can be solved in terms of a Meijer's function using [18, eq. (22)], with $a, b, c$, $d \in \mathcal{N}, \Psi, \varphi \in \Re^{*}$, and $H, J \in \Re$. Hence, after $N$ successive integrations, (A-2) can be expressed in closed form as in (5).

\section{REFERENCES}

[1] E. W. Stacy, "A generalization of the Gamma distribution," Ann. Math. Stat., vol. 33, no. 3, pp. 1187-1192, Sept. 1962.

[2] A. J. Coulson, A. G. Williamson, and R. G. Vaughan, "Improved fading distribution for mobile radio," IEE Proc. Commun., vol. 145, no. 3, pp. 197-202, June 1998.

[3] M. D. Yacoub, "The $\alpha-\mu$ distribution: A general fading distribution," in Proc. IEEE International Symposium on Personal, Indoor, Mobile Radio Commun., vol. 2, Lisbon, Sept. 2002, pp. 629-633.

[4] V. A. Aalo, T. Piboongungon, and C.-D. Iskander, "Bit-error rate of binary digital modulation schemes in generalized Gamma fading channels," IEEE Commun. Lett., vol. 9, no. 2, pp. 139-141, Feb. 2005.

[5] M. K. Simon and M.-S. Alouini, Digital Communication over Fading Channels, 2nd ed. New York: Wiley, 2005.

[6] N. C. Beaulieu and A. A. Abu-Dayya, "Analysis of equal gain diversity on Nakagami fading channels," IEEE Trans. Commun., vol. 39, no. 2, pp. 225-234, Feb. 1991.

[7] J. Cheng, C. Tellambura, and N. C. Beaulieu, "Performance of digital linear modulations on Weibull slow-fading channels," IEEE Trans. Commun., vol. 52, no. 8, pp. 1265-1268, Aug. 2004.

[8] G. K. Karagiannidis, D. A. Zogas, N. C. Sagias, S. A. Kotsopoulos, and G. S. Tombras, "Equal-gain and maximal-ratio combining over nonidentical Weibull fading channels," IEEE Trans. Wireless Commun., vol. 4, no. 3, pp. 841-846, May 2005.

[9] T. Piboongungon, V. A. Aalo, and C.-D. Iskander, "Average error rate of linear diversity reception schemes over generalized gamma fading channels," in Proc. IEEE Southeastcon, Ft. Lauderdale, FL, Apr. 2005, pp. $265-270$.

[10] N. C. Beaulieu, "An infinite series for the computation of the complementary probability distribution function of a sum of independent random variables and its application to the sum of Rayleigh random variables," IEEE Trans. Commun., vol. 38, no. 10, pp. 1463-1474, Sept. 1990.

[11] C. W. Helstrom, "Performance of receivers with linear detectors," IEEE Trans. Aerosp. Electron. Syst., vol. 26, no. 2, pp. 210-217, Mar. 1990.

[12] _ _ "Computing the distribution of sums of random sine waves and of Rayleigh-distributed random variables by saddle-point integration," IEEE Trans. Commun., vol. 45, no. 11, pp. 1487-1494, Nov. 1997.

[13] J. C. S. S. Filho and M. D. Yacoub, "Nakagami- $m$ approximation to the sum of $m$ non-identical independent Nakagami- $m$ variates," Electron. Lett., vol. 40, no. 15, pp. 951-952, July 2004.

[14] J. Hu and N. C. Beaulieu, "Accurate closed-form approximations to Rayleigh sum distributions and densities," IEEE Commun. Lett., vol. 9, no. 2, pp. 109-111, Feb. 2005.

[15] G. K. Karagiannidis, T. A. Tsiftsis, and N. C. Sagias, "A closedform upper-bound for the distribution of the weighted sum of Rayleigh variates," IEEE Commun. Lett., vol. 9, no. 7, pp. 589-591, July 2005.

[16] I. S. Gradshteyn and I. M. Ryzhik, Table of Integrals, Series, and Products, 6th ed. New York: Academic, 2000.

[17] J. Cheng and T. Berger, "Performance analysis for MRC and postdetection EGC over generalized Gamma fading channels," in Proc. IEEE Wireless Commun. and Networking Conf., vol. 1, New Orleans, LA, Mar. 2003, pp. 120-125.

[18] V. S. Adamchik and O. I. Marichev, "The algorithm for calculating integrals of hypergeometric type functions and its realization in REDUCE system," in Proc. International Conf. on Symbolic and Algebraic Computation, Tokyo, Japan, 1990, pp. 212-224.

[19] M. Abramovitz and I. A. Stegun, Handbook of Mathematical Functions with Formulas, Graphs, and Mathematical Tables, 9th ed. New York: Dover, 1972.

[20] G. K. Karagiannidis, "Moments-based approach to the performance analysis of equal gain diversity in Nakagami- $m$ fading," IEEE Trans. Commun., vol. 52, no. 5, pp. 685-690, May 2004.
[21] S. Sampei, Applications of Digital Wireless Technologies to Global Wireless Communications. London: Prentice Hall, 1997.

[22] G. K. Karagiannidis, T. A. Tsiftsis, and R. K. Mallik, "Bounds of multihop relayed communications in Nakagami- $m$ fading," IEEE Trans. Commun., vol. 54, no. 1, pp. 18-22, Jan. 2006.

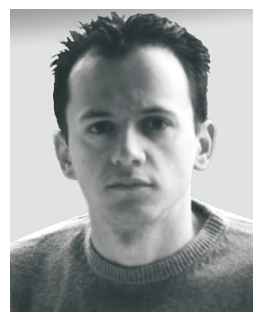

Nikos C. Sagias (S'03-M'05) was born in Athens, Greece in 1974. He received the B.Sc. (in Physics) in 1998, the M.Sc. (in Electronics \& Telecommunications) in 2000, and the Ph.D. (in Telecommunications) in 2005, degrees from the Department of Physics (DoP), University of Athens (UoA), Greece. Since 2000, he has cooperated with the Laboratory of Electronics of the DoP/UoA and the Institute for Space Applications and Remote Sensing of the National Observatory of Athens, where he has participated in several national and European R\&D projects. Currently, he is with the National Centre for Scientific Research "Demokritos," where he is a research associate at the Institute of Informatics and Telecommunications.

Dr. Sagias has authored or co-authored more than 20 journal and 15 conference papers. He acts as a reviewer for several international journals (including IEEE Transactions on Wireless Communications, IEEE Transactions on Communications, IEEE Transactions on Vehicular Technology, IEEE Communications Letters, and IEE Electronics Letters) and conferences, and he is the recipient of an Ericsson Award for his Ph.D. thesis. His current research interests include topics as wireless telecommunications, diversity receivers, fading channels and information theory. He is a member of the IEEE and the Hellenic Physicists Association.

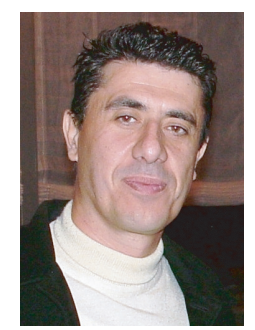

George K. Karagiannidis (S'95-M'97-SM'04) was born in Pithagorion, Samos Island, Greece. He received his university degree in 1987 and his Ph.D degree in 1999, both in Electrical Engineering, from the University of Patras, Patras, Greece.

From 2000 to 2004 he was Researcher at the Institute for Space Applications and Remote Sensing, National Observatory of Athens, Greece. In June 2004, he joined the faculty of Aristotle University of Thessaloniki, Greece where he is currently an Assistant Professor at the Electrical and Computer

Engineering Department.

His major research interests include wireless communications theory, digital communications over fading channels, satellite communications and freespace optical communications. He has published and presented more than 70 technical papers in scientific journals and international conferences, he is co-author in two chapters in books and also co-author in a Greek Edition Book on Mobile Communications.

Dr. Karagiannidis acts as reviewer for several international journals and he served as Technical program Committee Member for ICC'04 and ICC'05. $\mathrm{He}$ is member of the Editorial Boards of IEEE Communications Letters and EURASIP Journal on Wireless Communications and Networking. 


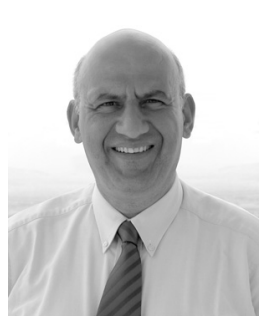

P. Takis Mathiopoulos (SM'94) is currently Director of Research at the Institute for Space Applications and Remote Sensing (ISARS) of the National Observatory of Athens (NOA), where is has established the Wireless Communications Research Group. As ISARS' Director he has lead the Institute to a significant expansion, $\mathrm{R} \& \mathrm{D}$ growth and international scientific recognition. For these achievements, ISARS has been selected as one of the national Centers of Excellence for the years 20052008. Before joining ISARS, he worked in the early 80's at Raytheon Canada Ltd. in the areas of air-navigational and satellite communications. In the late 80's he joined the Department of Electrical and Computer Engineering (ECE) of the University of British Columbia (UBC), where he was a faculty member for 14 years last holding the rank of Full Professor. Maintaining his ties with academia, he is an adjunct Professor of ECE at UBC and also is teaching part-time at the Department of Informatics and Telecommunications, University of Athens.

Over the years, Prof. Mathiopoulos has supervised university and industry based R\&D groups and has successfully acted as technical manager for large Canadian and European R\&D projects. He has been the PI for more than 25 research grants and has supervised the theses of 30 graduate students. His research contributions include original research work in the areas of optimal communications over fading channels, channel characterization and measurements, advanced coding techniques, including turbo-codes, diversity and synchronization, HDTV, neural networks, smart antennas, UMTS and SUMTS, software radios, MIMOs and UWB. Together with Prof. D. Makrakis of the University of Ottawa, they have published a paper in GLOBECOM'89 establishing for the first time in the open technical literature the link between MLSE and multiple differential detection for the AWGN and fading channels.
His publication record includes close to 150 papers in journals and international conference proceedings, about 50 of which have been published in IEEE journals. He is on the Editorial board of several scientific journals, including the IEEE Transactions on Communications. He has regularly acted as a consultant for various governmental and private organizations. Since 1993, he has served on a regular basis as scientific advisor and technical expert for the European Commission (EC) for the ACTS and IST programs. In this capacity, he has been appointed by the EC in numerous high level advisory, evaluation and auditing panels in the technical areas of telecommunications, information technology and electronic commerce and publishing. He has been a member of the TPC of more than 50 international conferences and has served as Vice Chair for the IEEE VTC2006-S. Prof. Mathiopoulos has delivered numerous invited presentations, including plenary lectures, and has taught many short courses all over the world.

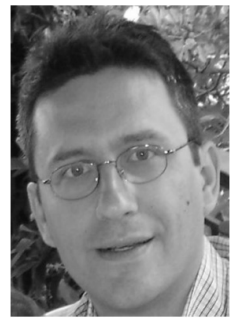

Theodoros A. Tsiftsis (S'02-M'04) was born in Lamia, Greece, in 1970. He received the B.Sc. degree in Physics from the Aristotle University of Thessaloniki, Greece, in 1993, and the M.Sc. degree in Digital Systems Engineering from the HeriotWatt University, Edinburgh, Scotland, U.K., in 1995. Also, he received the M.Sc. degree in Decision Sciences from the Athens University of Economics and Business, Greece, in 2000. He is currently working towards the Ph.D. degree in Electrical Engineering at the University of Patras, Greece. He is also affiliated as a research associate with the Institute of Space Applications and Remote Sensing (ISARS), National Observatory of Athens (NOA), where he is participating in various $R \& D$ projects. His research interests are digital communications over fading channels, multihop wireless communications and free-space optical communication systems. 